\title{
Research on the Key Technology of Medium Voltage Environmental Protection Metal-enclosed Switchgear
}

\author{
WANG Ning*, XING Lin, WU Peng, WANG Suo, HU Yuan \\ ${ }^{1}$ State Grid Hebei Economic Research Institute, Shijiazhuang, Hebei, 050000, China
}

\begin{abstract}
This article introduces environmentally-friendly metal-enclosed switchgear, and comprehensively summarizes the development status, technical characteristics, technical difficulties and development trends of solid-insulated ring network cabinets and environmentally-friendly gas-insulated switch cabinets. The development status of solid insulated ring network cabinets and environmentallyfriendly gas insulated switch cabinets are reviewed, and their technical characteristics are summarized in terms of volume, structure, maintainability, safety and environmental adaptability. Focus on the analysis and study of the technical difficulties of solid insulated ring network cabinets and environmentally friendly gas insulated switch cabinets, and pointed out the partial discharge control of the surface of the insulating parts, the strengthening of the sealing, the cost control, the recycling and utilization of epoxy resin, the optimization of insulation, and the breaking capacity. The guarantee of the quality, the selection and recovery of mixed gas are their future development trends. It provides a useful reference for the development and improvement of environmentally-friendly metal-enclosed switchgear.
\end{abstract}

\section{Introduction}

Medium-voltage switchgear is widely used in the power distribution system of the power grid and the majority of medium-voltage users. It is an indispensable product in a strong and smart grid, which is directly related to the safe and reliable operation of the grid.

Since the 1950 s, the development of medium voltage switchgear has gone through the stages of open type, box type, and metal enclosed type. There is no problem in meeting the functional requirements of the equipment, and the products have already been serialized and have full coverage. At present, innovation and technological progress are constantly unfolding around the market and the needs of society and the times of safety, reliability, miniaturization, and environmental protection of switchgear. In order to protect the global environment, SF6 was listed as a greenhouse gas in the Kyoto Protocol in 1997. Among them, CO2 has the largest impact, accounting for more than $60 \%$, and SF6 is $0.1 \%$. Although its impact is small at present, it is potentially harmful. Because a molecule of SF6 has 23900 times the greenhouse effect of a $\mathrm{CO} 2$ molecule, and the decay period of SF6 molecule is very long, about 3200 years. About $50 \%$ of SF6 produced globally is used in the power industry, and $80 \%$ of this is used in switchgear.

\section{Definition and classification of environmentally friendly metal enclosed switchgear}

When it comes to environmental protection, people usually think of reducing greenhouse gas emissions. In fact, this is just one of the many indicators of environmental protection. Environmental protection is a relative concept. Environmentally friendly switchgear needs to be judged not only to prevent the global greenhouse effect, but also to make a comprehensive judgment from some other environmental protection indicators. For example, reducing or reducing the use of toxic and hazardous substances, recycling of material resources (such as minimizing the use of thermosetting materials), reducing the energy required for target activities (energy saving), reducing the weight and volume of products (saving materials and land), The reuse after the nominal life of the product, the recycling capacity and energy consumption of recycled materials, etc., are indicators of the environmental impact of the product throughout its life cycle. In addition, the environmental adaptability of the switchgear should also be considered. It is mainly reflected in the fact that it is not easily affected by the surrounding environment, including pollution, heat and humidity, salt spray, high altitude and air pollution.

Therefore, environmentally-friendly metal-enclosed switchgear can be temporarily defined as 'switchgear with little or no use of SF6 that has little impact on the 
environment during the product's life cycle and has environmental adaptability'. Specifically, it can be divided into three categories: solid insulated switchgear (SIS), gas insulated switchgear that does not use SF6 (such as dry air insulated switchgear or N2 insulated switchgear), and gas insulated switchgear that rarely uses SF6, that is, inflatable Pressure and SF6 charge ratio should be restricted (for example, SF6 and N2 mixed gas insulated switchgear).

Environmental protection metal enclosed switchgear products are mainly divided into two categories:

(1) Ring network switchgear - rated current 630A and below, breaking current 20-25kA and below;

(2) General switchgear-rated current 630A and above, breaking current $25 \mathrm{kA}$ and above.

\section{The development status and technical characteristics of environment-friendly metal-enclosed switchgear}

\subsection{Solid insulated ring network cabinet}

The insulation and arc extinguishing of the switch body of the solid insulation ring network cabinet adopts vacuum medium, and the external insulation adopts solid material, and each circuit is connected by an insulated closed bus. The insulating cylinder uses solid-sealed pole technology to organically combine the vacuum interrupter, the main conductive circuit and the insulating support into a whole. It can realize a fully insulated, fully sealed and maintenance-free structure, with a smaller volume, a unit structure that can be dismantled and replaced, and flexible expansion.

The insulating cylinder is made of epoxy resin, and the bus bar is encapsulated with silicon rubber. This structure not only has good environmental adaptability, but also fundamentally solves the impact of SF6 gas expansion in high temperature areas. Therefore, it is especially suitable for applications in high temperature areas. The solid insulated ring network cabinet eliminates the pressure gas box components, avoids the problem of gas box deformation and deflation in high altitude areas, and is suitable for applications in plateau areas.

\subsection{Environmental protection gas insulated switchgear}

Medium-pressure gas-insulated metal-enclosed switchgear is also referred to as C-GIS. The feature of CGIS is to seal all high-voltage components in a boxshaped container, and the box is filled with insulating gas of lower pressure (0.02 0.05MPa gauge pressure). Generally, a vacuum interrupter is used to break the rated current and short-circuit current. With technological progress, C-GIS is moving towards the use of mixed gas, dry air, N2, CO2 and other insulating gases. Use as little or no SF6 gas as possible. At present, some Japanese manufacturers such as Hitachi, Meidensha, and Fuji Electric have implemented dry air insulation products on $24 \mathrm{kV}, 36 \mathrm{kV}$ and $72 \mathrm{kV}$ equipment. In China, there are three main categories of environmentally friendly gas insulated switchgear: the first is to charge clean and dry air, which is mainly used in $12 \sim 40.5 \mathrm{kV}$ products; the second is to charge $\mathrm{N} 2$ or $\mathrm{CO} 2$, which is mainly used in $12 \mathrm{kV}$ products; the third is It is filled with SF6 mixed gas. Studies have shown that $20 \%$ SF6 and $80 \%$ N2 mixed gas have good insulation performance, $10 \% \mathrm{SF} 6$ and $90 \% \mathrm{~N} 2$ mixed gas have been used in $24 \mathrm{kV}$ products, SF6 and N2 each account for $50 \%$ of the mixed gas It has been applied to $40.5 \mathrm{kV}$ products.

\subsection{Comparative Study on Insulation Performance of Nitrogen, Dry Air and SF6}

In a slightly uneven electric field, the electrical strength of SF6 is 2.5 to 3 times that of dry air and nitrogen. The breakdown electric field strength Eb of SF6 or a mixture of SF6 and nitrogen has been well simulated and is widely used in low pressure and high pressure gas insulation design.

The electric strength of nitrogen is basically the same as that of air. The rod-rod and rod-plate electrodes are used for power frequency breakdown test under rated atmospheric pressure. The deviation of nitrogen relative to air is as follows: the deviation of the test electrode rod diameter is $10-60 \mathrm{~mm}$, and the deviation of the net distance between electrodes is $10-100 \mathrm{~mm}$. The test results show that the deviation of the relative electric strength of nitrogen relative to air can be $0.85 \sim 1.25$. Therefore, when designing the nitrogen-insulated ring network cabinet, the breakdown electric field strength can be calculated based on the empirical formula of the air breakdown electric field strength, and the deviation 0.85 is taken as the insulation margin coefficient. Estimating the breakdown electric field strength $\mathrm{Eb}$ between the cylinders and the cylinder-to-board common in the switchgear under the condition of air insulation, namely the empirical formula:

$$
E_{b}=30.3 \delta\left(1+\frac{0.298}{\sqrt{r \delta}}\right)
$$

In the formula: $\mathrm{Eb}$ is the breakdown electric field strength, in $\mathrm{kV} / \mathrm{m} ; \mathrm{r}$ is the radius of the electrode, in $\mathrm{m} ; \delta$ is the relative gas density relative to standard atmospheric conditions (temperature is $20^{\circ} \mathrm{C}$ and pressure is $101.325 \mathrm{kPa}$ ).

The high-voltage insulation design test device is shown in Figure 1. The test is filled with different air pressures into the air box, and the power frequency ( 1 minute) and lightning impulse $(1.2 / 50 \mu \mathrm{s})$ withstand voltage tests are performed. The test electrode is shown in Figure 2. During the research, the distance between the electrodes can be changed. The insulation performance of $\mathrm{N} 2$, dry air and $\mathrm{CO} 2$ is tested under different air pressure conditions within the range of $0 \sim 0.3 \mathrm{MPa}$ (the relative value under the ambient temperature of $\left.20^{\circ} \mathrm{C}\right)$. The power frequency $(50 \mathrm{~Hz})$ and lightning impulse $(1.2 / 50 \mu \mathrm{s})$ test breakdown withstand voltage results are shown in Figure 3 and Figure 4 respectively. 


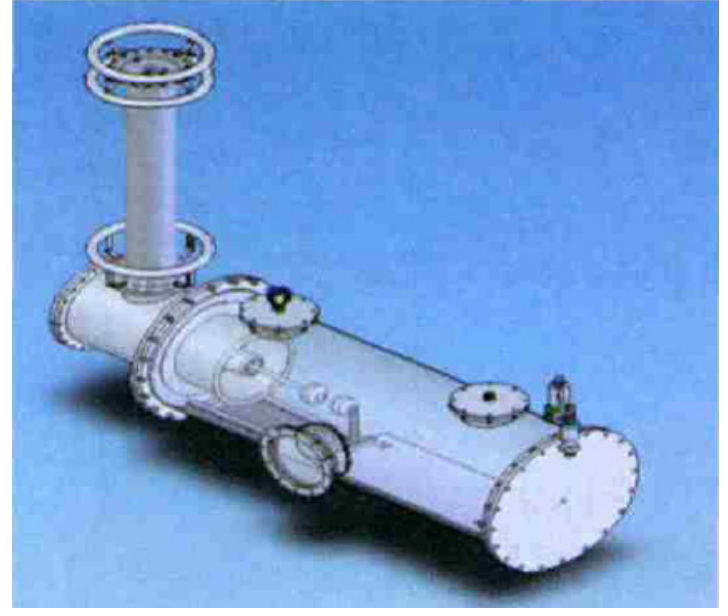

Figure 1 Schematic diagram of test device

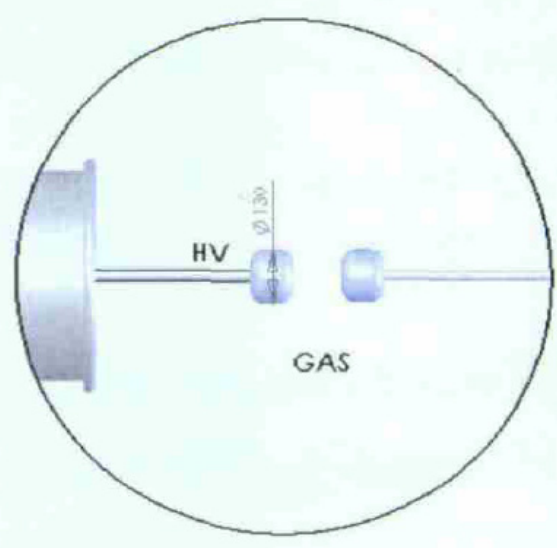

Figure 2 Schematic diagram of test electrode

It can be seen from the test results of Figure 3 and Figure 4 that the insulation performance of the three gases increases with the increase of gas pressure. The insulation performance of dry air is higher than that of nitrogen and $\mathrm{CO} 2$. Especially when the pressure reaches above $0.05 \mathrm{MPa}, \mathrm{CO} 2$ has the lowest insulation performance among the three gases. The conclusion of the comparison between dry air and nitrogen: the presence of oxygen has a positive effect on the insulation performance. Under lower air pressure $(0 \sim 0.05 \mathrm{MPa})$, the lightning impulse withstand voltage of $\mathrm{CO} 2$ is slightly higher than that of dry air and nitrogen. Based on the above research results, considering the greenhouse effect of $\mathrm{CO} 2$ gas, the use of clean dry air or nitrogen as the insulating medium meets environmental protection requirements, and the insulation design of the switchgear can refer to the above test results.

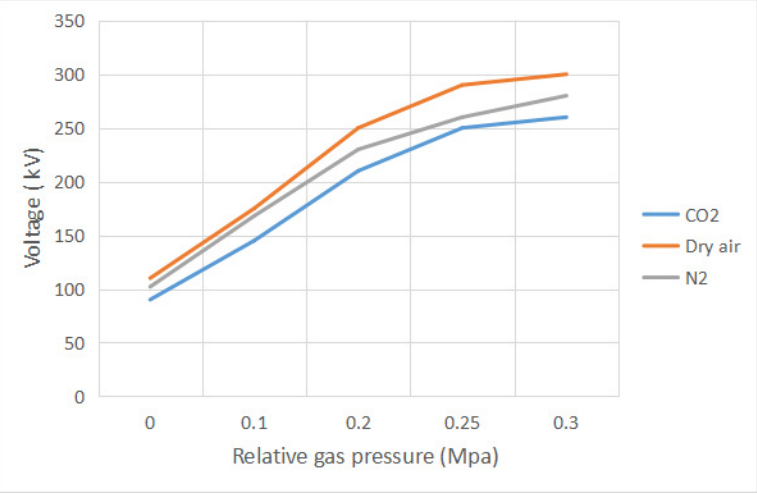

Figure 3 Power frequency withstand voltage characteristic curve (electrode distance: $70 \mathrm{~mm}$ )

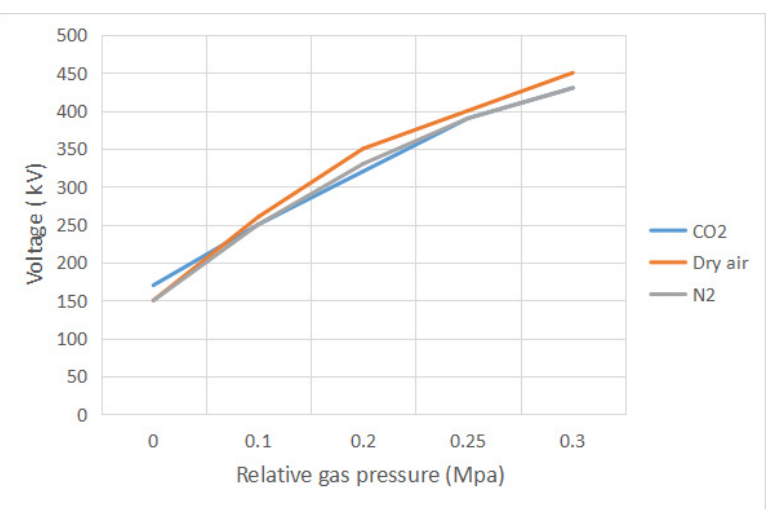

Figure 4 Lightning impulse withstand voltage curve (electrode distance: $70 \mathrm{~mm}$ )

It can be seen that SF6 is a strongly electronegative gas, and its molecules have a strong ability to absorb electrons and have high electrical strength. Compared with SF6 gas, the breakdown electric field strength of nitrogen is low. Using nitrogen as the gas insulation medium, the electric strength of the air gap and the insulation along the surface of the ring network cabinet is much lower than that of SF6. Therefore, if the nitrogen insulated ring network cabinet does not increase the size of the cabinet and the gas pressure, if it is to obtain the same insulation level as the SF6 insulated ring network cabinet, the insulation structure design must be optimized to reduce the air gap and the maximum solid insulation surface. Electric field strength.

\subsection{Advantages of environmentally friendly gas insulated switchgear}

In addition to environmental protection, the advantages of environmentally friendly gas insulated switchgear are mainly reflected in the following aspects:

(1) Small size. The environmentally friendly gasinsulated cabinet retains the advantages of gas-insulated switchgear in size, and the cabinet size is small, saving land;

(2) The amount of maintenance is small. Highvoltage insulation is not affected by the external environment and is not easy to age. Users do not need to deal with gas on site, which can reduce the life cycle cost of the equipment; 
(3) High modularity. Flexible combination and convenient splicing;

(4) Safe and reliable. All high-voltage components are sealed in a gas-filled metal shell, and the metal shell is reliably grounded to ensure the safety of operation and maintenance personnel.

\section{Technical difficulties and development trends of environmentally friendly metal-enclosed switchgear}

\subsection{Partial discharge control of solid insulated ring network cabinet}

The main problems to be solved in the design and production of solid insulated ring network cabinets are the continuity of shielding and grounding, the surface metalization of insulating materials, and the optimization of electric fields. The purpose is to coat the outer surface of the coated insulating medium with a conductive or semi-conductive shielding layer to make it grounded reliably, while reducing the impact of the use environment on the product. At present, a large part of the insulating surface of the existing domestic products has not been shielded and metalized, and the partial discharge index is unstable. Once a solid insulation discharge occurs, the insulation cannot be restored. As the discharge continues to increase, insulation breakdown and phase-to-phase short-circuit faults will occur. At the same time, because the surface of the equipment is not reliably grounded, personal injury or death accidents may occur when people touch it.

Therefore, it is necessary to start with the principle of solid insulation technology, scientifically calculate and optimize the design of the electric field, magnetic field, temperature field, shielding, and surface grounding of insulators, and strengthen the monitoring of partial discharge.

\subsection{Recycling of epoxy resin}

Epoxy resin is used as the main insulating material of solid insulated ring main unit, and its production process and disposal after scrap will produce toxic substances. At present, there are still controversies about the environmental protection of epoxy materials, and there is no technical breakthrough in the degradation treatment of epoxy materials.

Therefore, the development of high-performance epoxy resins and the realization of recyclability and reuse are issues that urgently need to be studied. It is understood that due to the long-term power frequency vibration of the solid insulation ring network cabinet put into operation in South Korea for many years, the solid insulation cracking or increased partial discharge has begun to cause frequent accidents. In-depth understanding, communication and reference should be carried out as soon as possible.

\subsection{Optimization of breaking capacity of environmentally friendly gas insulated switchgearRecycling of epoxy resin}

For the load switch in the ring main unit, the breaking capacity will be affected after the environmental protection gas is used. Using a vacuum interrupter to enhance the breaking capacity is a mature alternative. However, it is difficult to accept the vacuum interrupter as the isolation fracture. The vacuum fracture may have non-retentive breakdown discharge (NSDD) under the action of high voltage, and the cold welding phenomenon of the vacuum contact can easily lead to a drop in the impact level, which directly threatens personal safety. Therefore, the existing vacuum load switch needs to be matched with a three-station isolation/grounding switch, which not only has high cost, but also has a large product size.

Therefore, it is necessary to study a new type of switch structure so that the environmentally friendly gas can be used as an insulating medium and an arc extinguishing medium.

\subsection{Temperature rise and heat dissipation of environmentally friendly gas insulated switchgear}

The characteristic of gas-insulated switchgear is to seal the high-voltage components in a stainless steel gas box. The closed gas box can only dissipate the heat generated by the load current through limited convection, conduction and radiation. Therefore, the design current density of the gas-insulated switchgear Relative to air insulation needs to be appropriately reduced. The same design of different gases has a big difference in temperature rise margin. Under the same conditions, the difference between SF6 and N2 or dry air is more than 10K. Since the enclosed environment in the gas box of the gas insulated product will not be disturbed by the surrounding environmental factors, the contact resistance is stable, and the measures to increase the rated current to ensure the temperature rise of the load directly affect the increase in product material costs.

\section{Conclusion}

In summary, the solid insulated ring network cabinet has the characteristics of small size, maintenance-free, flexible structure, and strong environmental adaptability, and is especially suitable for use in high temperature and plateau areas. The partial discharge control of the surface of the insulating parts, the strengthening of the seal, the cost control, and the recycling of epoxy resin are its future technological trends. In addition to being environmentally friendly, the environmentally-friendly gas-insulated switchgear also has the advantages of small size, low maintenance, high modularity, safety and reliability. The technical difficulties are mainly embodied in the aspects of insulation optimization, guarantee of breaking capacity, temperature rise and heat dissipation, and selection and recovery of mixed gas. 
The environmentally friendly insulated switchgear does not use SF6 and its charging device, has no toxic arc decomposition products, is convenient to maintain, and does not require an insulating gas recovery device, thus reducing the monitoring project and operation and maintenance workload; the environmentally friendly insulated switchgear is a maintenance-free product, Not affected by atmospheric conditions, the product is not only environmentally friendly. Moreover, the area is small, and the product is safe and reliable. At present, environmentally friendly gas insulated switchgear has accumulated some operating experience and performance, and will occupy an important position in the medium voltage field in the future.

\section{References}

1. Chen Bo, $\mathrm{Xu}$ Aidong. Research status and development trend of environmental protection gas insulated metal enclosed switchgear. J. Science and Technology Innovation and Application, 2017(03)

2. Cui Wenjun, Zhang Jiaosuo. The use of dry air as an insulating medium in non-SF6 gas insulated switchgear. J. High-voltage electrical appliances, 2016(12)

3. $\mathrm{Xu}$ Hui. Good prospects for environmentally friendly gas insulated metal enclosed switchgear. J. Electrical Industry, 2015(05)

4. Qian Lixiao, Chen Shenyan. Development of $12 \sim 24 \mathrm{kV}$ nitrogen insulated ring network cabinet. J. High Voltage Technology,2014(12)

5. Tang Xin, Liao Sijun. Research progress of SF6 hybrid/substitution gas insulation performance. J. Insulating materials.,2014(06)

6. Sun Zhusen, Lan Jian. Technical characteristics and development trend of environmentally-friendly metal-enclosed switchgear. J. China Electric Industry (Technical Edition), 2014(06)

7. Zhang Jiaosuo. Realization and key technology of medium voltage environmentally friendly gas insulated switchgear. J. China Electric Power (Technical Edition), 2014(06) 